\title{
ORIGINAL
} ARTICLES

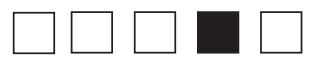

\section{Breastfeeding Education in Family Medicine Residencies: A 2019 CERA Program Directors Survey}

\author{
Zeynep Uzumcu, MD; Mary Beth Sutter, MD; Peter F. Cronholm, MD, MSCE
}

\begin{abstract}
BACKGROUND AND OBJECTIVES: Breastfeeding counseling is an integral skill for all family physicians, regardless of whether they ultimately practice maternal child health $(\mathrm{MCH})$. Evidence on time spent in breastfeeding education and resident competence is lacking. This study aimed to identify program characteristics associated with an increase the amount of breastfeeding education and program directors' (PDs) perceived competence of residents' breastfeeding counseling skills.
\end{abstract}

METHODS: A national survey of family medicine PDs including breastfeeding questions was conducted as part of the 2019 CERA survey. We specifically sought to identify variables that correlate with increased breastfeeding education time and perceived competence.

RESULTS: Family medicine programs with greatest breastfeeding education time and perceived resident competence included lactation consultants, had more $\mathrm{MCH}$ visits in resident continuity clinic, more graduates that practice $\mathrm{MCH}$, and included competency evaluations by faculty. There was more volume of breastfeeding education in programs with group prenatal care and an academic affiliation. There was greater perceived competence among programs with more hours of breastfeeding training overall.

CONCLUSIONS: This study defines associations with curricular targets for improved breastfeeding counseling competence among family medicine residents. Inclusion of lactation consultants, regular faculty observation of counseling skills, and group prenatal care may be gradually introduced in programs to strengthen resident education and skills in breastfeeding counseling. The body of evidence in this field remains lacking, and further research is needed to characterize curricular interventions that increase resident competence in this important skill.

(Fam Med. 2020;52(7):497-504.)

doi: 10.22454/FamMed.2020.579473

amily physicians are uniquely poised to provide breastfeeding counseling as a part of standard prenatal, intrapartum, and postpartum care. Breastfeeding knowledge is a valuable skill for all family physicians, regardless of whether or not they choose

to practice obstetrics, related to their ongoing interface with families at all stages of development. Evidence supports the positive maternal and child health outcomes of breastfeeding, and demonstrates its wider social and economic benefit. ${ }^{1}$ National professional organizations including the
American Academy of Family Physicians, ${ }^{1}$ the United States Preventive Services Task Force, ${ }^{2}$ the American Academy of Pediatrics, ${ }^{3}$ Healthy People 2020, ${ }^{4}$ the World Health Organization, ${ }^{5}$ and the US Surgeon General,${ }^{6}$ have called for increased clinician training, recognizing the benefits of breastfeeding.

Few studies have examined the relationship between breastfeeding education and clinical competence in breastfeeding counseling. Two studies showed that half of surveyed family medicine, pediatric, and OBGYN practicing physicians rated their residency training in breastfeeding counseling as inadequate. ${ }^{7,8}$ A previous study described a curricular model implemented through didactic and interactive training of medical students and residents in pediatrics, OB-GYN and family medicine, that showed feasibility and positive reception by learners. ${ }^{9}$ The impact of a similar 2-week curricular intervention was examined for lactation education across family medicine, pediatric, and OBGYN residencies $(n=407)$, finding
From the Department of Family and Community Medicine, Jefferson University Hospital, Philadelphia, PA (Dr Uzumcu); Department of Family Medicine, Brown University, Providence, RI (Dr Sutter); and the Department of Family Medicine and Community Health, Center for Public Health Initiatives, Leonard Davis Institute of Health Economics, University of Pennsylvania, PA (Dr Cronholm). 
improvement in knowledge, practice patterns, and exclusive breastfeeding rates at 6-months (OR 4.1 [95\% CI: 1.8-9.7]). ${ }^{10} \mathrm{~A} 2017$ survey of residency program directors (PDs; combined $n=95$ ) found that the median total hours of breastfeeding education was 8 hours in family medicine, 23 hours over 4 years in OB-GYN, and 9 hours over 3 years for pediatrics. ${ }^{11}$ The study also found passive lecture to be the most common modality of teaching.

Innovation in clinical care in the peripartum period continues to evolve nationally, with the emergence of the WHO Baby-Friendly Hospital Initiative and the CenteringPregnancy prenatal care model. ${ }^{12,13}$ Studies of CenteringPregnancy show higher breastfeeding initiation rates compared with traditional care (66.5\% vs $54.6 \%) .{ }^{13}$ Currently, the extent to which these patient care models are used in continuity family medicine residency training sites and their impact on competency in breastfeeding counseling are poorly understood. Moreover, involvement of lactation consultants in resident education has been shown to positively impact resident competence and patient breastfeeding rates. ${ }^{14,15}$

This study aimed to identify program characteristics that were associated with the amount of breastfeeding education and program directors' (PDs) perceived competence of residents' breastfeeding counseling skills.

\section{Methods}

\section{Survey Hypotheses}

Our hypotheses for the survey addressed training setting, $\mathrm{MCH}$ volume in continuity clinics, involvement of lactation consultants in resident education, resident exposure to group prenatal care and baby-friendly institutions, presence of faculty evaluations, graduate $\mathrm{MCH}$ practice rates, and hours of breastfeeding education.

We hypothesized that academic and community-based programs would have no difference, and that population size would not affect the total amount of time for breastfeeding training. Using regional breastfeeding data from the CDC's National Immunization Survey, we hypothesized that Western (Pacific and Mountain) programs, which tend to produce more graduates that ultimately practice $\mathrm{OB}$, and where regional breastfeeding continuation rates are higher compared to other regions (ie, the Northeast), would have more time spent in breastfeeding training and a higher perceived competence of breastfeeding. ${ }^{16,17}$

We hypothesized that programs with more resident exposure to $\mathrm{MCH}$ continuity care would have more training and higher perceived competence in breastfeeding. We also hypothesized that training by lactation consultants and exposure to the WHO Baby-Friendly model of institutional breastfeeding promotion would be associated with increased curricular time for breastfeeding training and perceived competence. We hypothesized that programs with faculty involvement in evaluating resident breastfeeding counseling skills, and with more graduates who ultimately practice $\mathrm{MCH}$, would have more time dedicated to training and more perceived competence.

Finally, we expected that programs that dedicated more time to breastfeeding training would have greater perceived competence by PDs.

\section{Procedures}

The questions were part of a larger omnibus survey conducted by the Council of Academic Family Medicine Educational Research Alliance (CERA). The methodology of the CERA Program Directors Survey has previously been described in detail. ${ }^{18}$ The CERA Steering Committee evaluated questions for consistency with the overall subproject aim, readability, and existing evidence of reliability and validity. Pretesting was done on family medicine educators who were not part of the target population. Questions were modified following pretesting for flow, timing, and readability. The
American Academy of Family Physicians Institutional Review Board approved the project in May 2019. Data were collected from May 2019 to July 2019.

\section{Participants}

The sampling frame for the survey was all ACGME-accredited US family medicine residency PDs as identified by the Association of Family Medicine Residency Directors (AFMRD). Email invitations to participate were delivered with the survey utilizing the online program Survey Monkey. Seven follow-up emails to encourage nonrespondents to participate were sent after the initial email invitation.

\section{Outcome Measures}

The first outcome of interest was the measure of total breastfeeding educational time during residency, which was created by combining estimates of the number of hours devoted to didactic and interactive teaching during residency. The second outcome of interest was PDs' perception of resident skills in breastfeeding counseling. This was grouped as a dichotomous variable comparing residents who were rated as "very competent" and "expert" to those rated as "somewhat competent" and "poor." An additional outcome was programs' anticipated changes to the amount of time spent on breastfeeding training in the next year (dichotomized as "same" or "less training," versus "more training").

\section{CERA Demographic Data}

Independent variables of interest from the demographic data were program type, dichotomized as academic (university and university-affiliated) versus community programs (all nonuniversity program types), and rural service communities (dichotomized as communities of $\leq 30,000$ people compared to larger communities). We also examined Western programs (grouping Mountain and Pacific programs) dichotomized by comparing the combined group versus all other regions. Likewise, New 
England programs were dichotomized to compare the group to all other programs. Demographic data is included in Table 1.

\section{Study-Specific Variables}

We examined percentage of maternal child health $(\mathrm{MCH})$ visits as a continuous variable. The variable of resident training in baby-friendly hospitals was dichotomized to compare "all" or "some" residents learning in this setting to "no residents." Similarly, the variable of residents training in a CenteringPregnancy or group prenatal care model was dichotomized to compare "all" or "some" residents to "no" residents. Likewise, in-person resident training by a lactation consultants was dichotomized to compare "all" or "some" residents to "no" residents. Faculty providing evaluation of resident breastfeeding competence was dichotomized as "yes" versus "no." Finally, we examined graduates continuing $\mathrm{MCH}$ in their future practice as a continuous variable. The above questions included the possible answer of "unsure." Any questions that were answered as "unsure" were excluded from the analysis in this survey.

\section{Analysis}

Linear regression was used to model associations between independent variables and continuous outcomes. Logistic regression was used to compute odds ratios to estimate the associations between independent variables and dichotomized outcomes. We employed a level of statistical significance set at $\alpha=0.05$, recognizing that tests of statistical significance are approximations that serve as aids to interpretation and inference. We used Stata software (Stata Corp, College Station, TX) for analysis.

\section{Results}

Respondents

At the time of the survey, there were 624 PDs, of whom 16 opted out of CERA survey participation. The survey was emailed to 608 individuals; 18 emails were undeliverable. The general CERA survey was responded to by $45.4 \%$ of PDs (268/590).

Seventeen programs did not complete breastfeeding-specific survey items and were excluded from the present analyses leaving a final breastfeeding survey sample of $251 / 590$ (response rate of $42.5 \%$ ) for the analyzed data. For a summary of the results obtained, see Tables 2 and 3. Figures 1 and 2 depict the associations between program characteristics with hours of breastfeeding curricular time and perceived competence. Access to complete survey results is availabe through the CERA 2019 Residency Program Directors (PD15) Survey Results Clearinghouse. $^{19}$

\section{Hours of Passive and Active \\ Breastfeeding Training}

The hours of passive didactic training and interactive breastfeeding training are presented in Table 4, and show that residents receive

Table 1: Demographic Characteristics of Programs Surveyed

\begin{tabular}{|c|c|}
\hline Variables & $\begin{array}{c}\text { Percent of } \\
\text { Respondents }\end{array}$ \\
\hline \multicolumn{2}{|l|}{ Program Type } \\
\hline University-based & 16.9 \\
\hline Community-based, university-affiliated & 60.1 \\
\hline Community-based, nonaffiliated & 18.2 \\
\hline Military & 1.6 \\
\hline Other & 3.2 \\
\hline \multicolumn{2}{|l|}{ Geographic Location } \\
\hline New England (NH, MA, ME, VT, RI, or CT) & 3.2 \\
\hline Mid-Atlantic (NY, PA, or NJ) & 14.9 \\
\hline South Atlantic (PR, FL, GA, SC, NC, VA, DC, WV, DE, or MD) & 13.7 \\
\hline East South Central (KY, TN, MS, or AL) & 6.1 \\
\hline East North Central (WI, MI, OH, IN, or IL) & 19.4 \\
\hline West South Central (OK, AR, LA, or TX) & 9.7 \\
\hline West North Central (ND, MN, SD, IA, NE, KS, or MO) & 10.9 \\
\hline Mountain (MT, ID, WY, NV, UT, AZ, CO, or NM) & 9.3 \\
\hline Pacific (WA, OR, CA, AK, or HI) & 12.9 \\
\hline \multicolumn{2}{|l|}{ Community Size } \\
\hline Less than 30,000 & 10.9 \\
\hline 30,000 to 74,999 & 20.6 \\
\hline 75,000 to 149,000 & 18.2 \\
\hline 150,000 to 499,00 & 22.6 \\
\hline 499,000 to 1 million & 14.9 \\
\hline More than 1 million & 12.9 \\
\hline \multicolumn{2}{|l|}{ Residents in Program } \\
\hline Less than 19 & 41.5 \\
\hline 19 to 31 & 40.3 \\
\hline Greater than 31 & 16.5 \\
\hline
\end{tabular}


Table 2: Coefficient of Relationship Program Traits and Reported Volume of Breastfeeding Training

\begin{tabular}{|l|c|c|}
\hline \multicolumn{1}{|c|}{ Program Traits } & $\begin{array}{c}\text { Time of Breastfeeding } \\
\text { Training (Coefficient) }\end{array}$ & 95\% Cl \\
\hline Lactation consultants * & 2.38 & $(1.88-2.87)$ \\
\hline Faculty evaluation * & 1.45 & $(0.88-2.02)$ \\
\hline Group prenatal care * & 1.13 & $(0.51-1.74)$ \\
\hline Greater MCH visits * & 0.70 & $(0.46-0.95)$ \\
\hline Academic program * & 0.67 & $(0.036-1.31)$ \\
\hline Greater graduates practicing MCH * & 0.42 & $(0.26-0.58)$ \\
\hline Baby friendly & 0.16 & $(-0.55-0.87)$ \\
\hline Located in Western states & 0.37 & $(-0.28-1.01)$ \\
\hline Located in New England * & 0.16 & $(0.05-2.98)$ \\
\hline Smaller community $(\leq 30 \mathrm{~K}$ residents)* & -1.20 & $(-2.07-0.32)$ \\
\hline
\end{tabular}

* Statistically significant findings, $P \leq .05$.

Table 3: Relationship Between Program Characteristics and Perceived Breast Feeding Counseling Competence

\begin{tabular}{|l|c|c|}
\hline \multicolumn{1}{|c|}{ Program Characteristics } & \multicolumn{2}{c|}{ Perceived Competence } \\
\hline Faculty evaluation * & Odds Ratio & 95\% Cl \\
\hline Lactation consultants * & 3.81 & $(1.95-7.43)$ \\
\hline Located in New England & 3.52 & $(1.89-6.56)$ \\
\hline Academic program & 3.17 & $(0.68-14.59)$ \\
\hline Baby friendly & 1.82 & $(0.86-3.82)$ \\
\hline More hours of training overall * & 1.88 & $(0.83-4.22)$ \\
\hline More graduates practicing MCH * & 1.56 & $(1.33-1.84)$ \\
\hline More MCH visits * & 1.51 & $(1.25-1.83)$ \\
\hline Group prenatal care & 1.44 & $(1.09-1.89)$ \\
\hline Located in Western states & 1.16 & $(0.59-2.24)$ \\
\hline Smaller community $(\leq 30 \mathrm{~K}$ residents $)$ & 0.64 & $(0.46-1.95)$ \\
\hline
\end{tabular}

* Statistically significant findings, $P \leq .05$.

more passive than active training. The median number of hours in passive training per year is 3 . The median number of hours in interactive training per year is 2 hours, and the median of cumulative time is 5 hours per year. More time spent in breastfeeding training overall was associated with greater perceived breastfeeding competence (OR 1.56 [95\% CI 1.33-1.84]).
Associations Between Program Characteristics and Training Time and Perceived Competence Academic settings were associated with more breastfeeding education, but there was no statistically significant relationship between perceived competency in breastfeeding counseling and academic or nonacademic settings. Additionally, there was a statistically significant negative relationship between programs located in communities with populations $\leq 30,000$ and hours of total breastfeeding training, but no difference between population size and perceived resident competency in breastfeeding counseling. Furthermore, there was a statistically significant positive relationship between New England programs and amount of education, but no statistically significant effect in perceived competence. For Western programs (Mountain and Pacific grouped together), there was no statistically significant correlation between region and volume of education or perceived competence.

Programs with a higher proportion of $\mathrm{MCH}$ visits within resident continuity clinic had more hours of breastfeeding education and higher perceived competence of breast feeding counseling. The involvement of lactation consultants in resident education increased hours of resident education in breastfeeding and improved perceived competence in breastfeeding counseling.

Our survey demonstrated a likely positive relationship between group prenatal care models and more breastfeeding education, but no statistically significant effect on perceived resident competence. There was a positive association between baby-friendly institutions and hours of education and perceived competence in breastfeeding counseling, though these were not statistically significant. Programs that included group prenatal care experiences for all or some of their residents were more likely to also include training with lactation consultants for all or some of their residents (OR 2.38, [1.3-4.34]).

Programs that required faculty evaluation of resident breastfeeding counseling competence were associated with both more breastfeeding training and greater perceived competence of breastfeeding counseling. There was also a positive relationship between programs that produce a greater proportion of graduates who ultimately practice $\mathrm{MCH}$ and their volume of breastfeeding training and the perceived breastfeeding 
Figure 1: Relationship Between Program Traits and Time of Breastfeeding Training

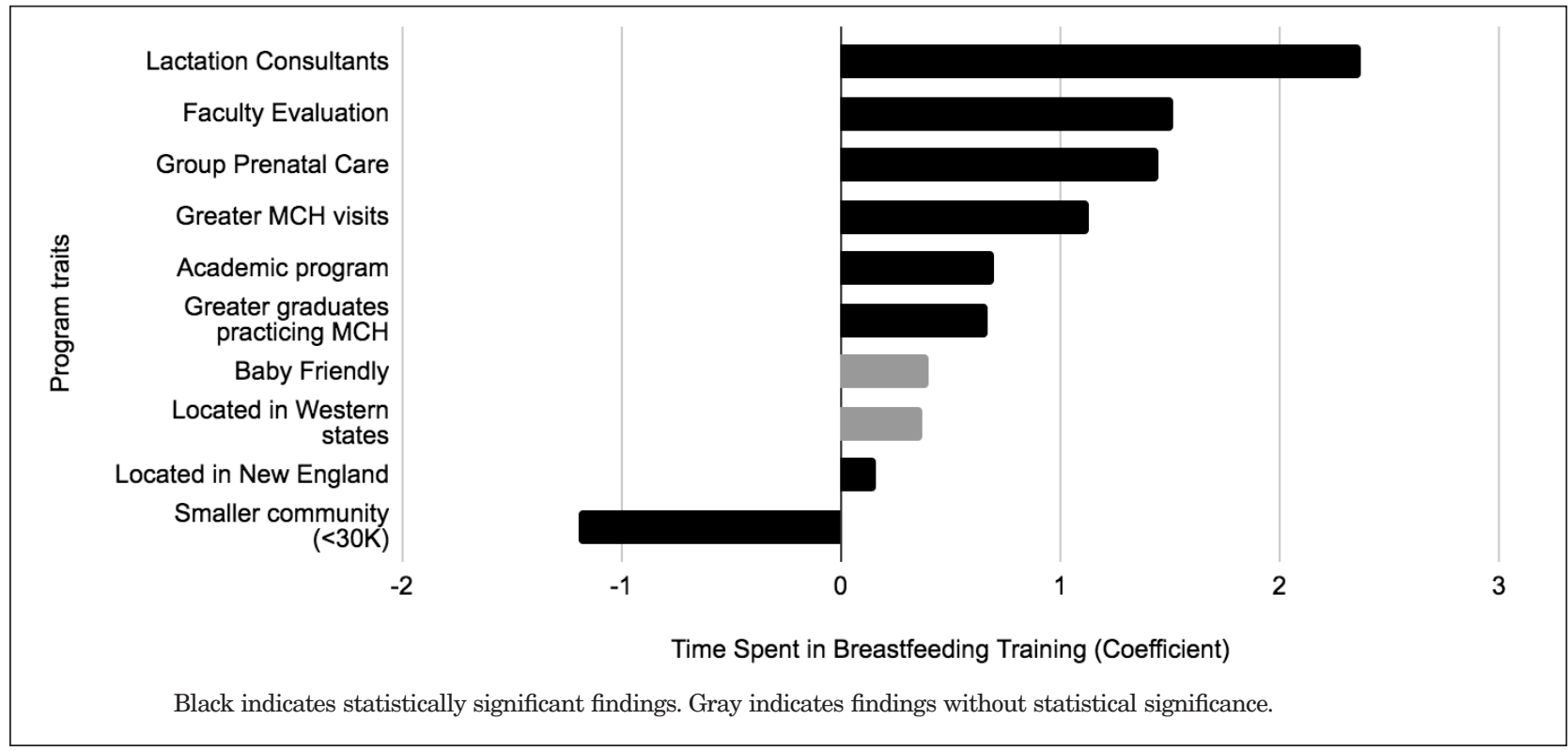

Figure 2: Relationship Between Program Traits and Perceived Breastfeeding Competence

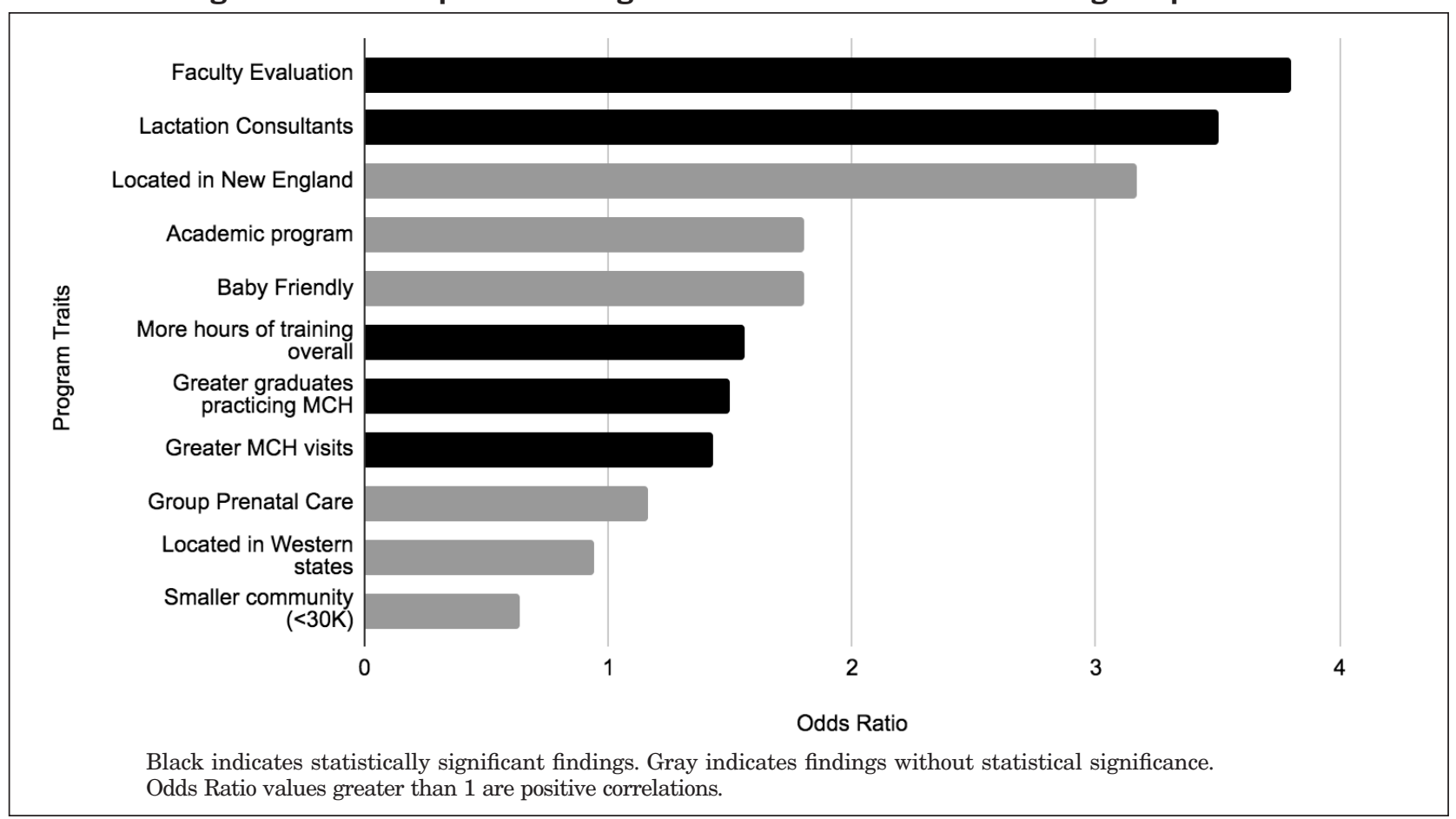

counseling competence of their graduates.

\section{Discussion}

Hours of Training

Compared to previous findings from a 2017 survey by Lien et al, our findings of 5 hours of median yearly training time is greater than their finding of 8 hours of training over 3 years for surveyed family medicine programs. ${ }^{11}$ Our findings are consistent with this study's previous findings, which showed that the majority of breastfeeding training occurs in the passive didactic setting.

\section{Training Setting}

Academic settings were associated with more resident education in breastfeeding, similar to studies of other MCH education topics. ${ }^{17}$ This may be related to more exposure to multidisciplinary teams in academic settings, and joint education of 
Table 4: Distribution of Hours of Passive and Active Breastfeeding Training

\begin{tabular}{|c|c|c|}
\hline $\begin{array}{c}\text { Passive Breastfeeding } \\
\text { Training (Hrs) }\end{array}$ & $\begin{array}{c}\text { Number of } \\
\text { Respondents }\end{array}$ & Percent of Respondents \\
\hline 0 & 8 & 3.33 \\
\hline 1 & 91 & 37.92 \\
\hline 2 & 79 & 32.92 \\
\hline 3 & 36 & 15.00 \\
\hline$\geq 4$ & 26 & 10.83 \\
\hline $\begin{array}{c}\text { Active Breastfeeding } \\
\text { Training (Hrs) }\end{array}$ & $\begin{array}{c}\text { Number of } \\
\text { Respondents }\end{array}$ & Percent of Respondents \\
\hline 0 & 60 & 25.42 \\
\hline 1 & 77 & 32.63 \\
\hline 2 & 56 & 23.73 \\
\hline 3 & 14 & 5.93 \\
\hline$\geq 4$ & 29 & 12.29 \\
\hline
\end{tabular}

multiple residency specialties. Rural programs had less resident breastfeeding education, which represents a deficiency that is concerning given the lack of overall maternal child health resources and lower breastfeeding rates in rural areas. Family physicians are an important resource for rural maternity care and adequate core education is essential. Interestingly, there was no correlation either with academic settings or rural settings with perceived competence, indicating that PDs may be overlooking breastfeeding education as an important educational endpoint, and providing evidence for enhancing breastfeeding education as an easy target for improvement.

Although there are regionally higher breastfeeding initation and continuation rates in the Western United States, there was no statistically significant association between programs in the Western United States regionality and breastfeeding education time or perceived competence compared to all other regions. ${ }^{17,19}$ This is an interesting disconnect between our data and the locations in the country where we expected more training and competence.

New England also has higher breastfeeding initiation and continuation rates compared to the rest of the country, though $\mathrm{MCH}$ practice in family medicine is lower in this region. ${ }^{14,15,16}$ The survey showed more training time in this region, which may correlate with regional breastfeeding trends. Of note, however, the data underrepresented New England, with only $3.2 \%$ of respondents from this region, and may have been underpowered to demonstrate an effect.

\section{Volume of MCH Visits}

Higher volume of $\mathrm{MCH}$ visits in continuity clinic was associated with higher resident breastfeeding education and perceived competence. This finding is likely reflective of the value of repetition in acquiring competence in clinical counseling skills, and may also reflect the general emphasis placed on $\mathrm{MCH}$ within programs as well as the availability of $\mathrm{MCH}$ faculty. Programs that do not have a robust $\mathrm{MCH}$ faculty could target resident education through collaboration with other specialites (obstetrics, midwifery, lactation, pediatrics) to increase number of $\mathrm{MCH}$ related visits.

\section{Interdisciplinary Interventions}

The positive relationship between involvement of lactation consultants and increased resident breastfeeding education and competence demonstrates the importance of collaboration for the benefit of patient care.
Lactation consultants should be involved in education whenever possible, just as residents would learn from dieticians, pharmacists, and other nonphysician health specialties. The benefit of their involvement in academic learning environments has been established in recent research that showed increases in breastfeeding among patients exposed to lactation consultants. ${ }^{17}$ Outpatient support for lactation consultation is a valuable tool for residency clinics, where time alloted to the physician can be short for a prenatal, postpartum, or well-baby visit.

\section{Group Prenatal Care and Baby- Friendly Institutions}

We hypothesized that exposure to the WHO Baby-Friendly model of institutional breastfeeding promotion in the critical 0-2 days of postpartum and neonatal care would have a positive correlation with resident breastfeeding training and competence. Our survey data, however, showed no association between the training of all or some residents within this setting. This finding could reflect the lack of communication and collaboration between hospital, nursing, financial, and educational goals, but also demonstrates an important and simple area for improvement. For example, some programs have incorporated the Baby-Friendly staff education requirements with interactive residency teaching, in order to strengthen the relationship between nurses, residents, faculty and lactation consultants for the greater good of maternity care. This lack of effect may also correlate with a recent report that Baby-Friendly institutions did not yield significant outcomes in breastfeeding continuation. ${ }^{20}$

We hypothesized that residencies in which all or some residents engaged in group prenatal care, there would be increased hours of breastfeeding education and increased perceived competence in breastfeeding counseling. Our survey data demonstrate a positive relationship between these variables. This finding may be due to the interactive peer to 
peer nature of group model, or may reflect a greater proportion of $\mathrm{MCH}$ patients in residency clinics with a group prenatal care model. Additionally, programs that support a group prenatal care model within resident continuity care may dedicate a greater focus on $\mathrm{MCH}$ education or have a higher proportion of faculty with $\mathrm{MCH}$ training, and also appear to involve lactation consultants more within resident education.

\section{Faculty Assessment}

The expectation of faculty assessment in resident breastfeeding counseling competence was associated with more training time and greater resident competence. Programs that dedicate time to resident evaluation in this skill likely recognize its importance to their graduates' practice. Alternately, this may represent a reporting bias, in that faculty groups that evaluated residents were also more aware of the extent of education and resident knowledge.

\section{Graduate Practice Characteristics} Our data support our hypothesis that programs with more graduates who ultimately practice maternal child health (prenatal, postpartum, newborn care) also provide more hours of lactation education and have more residents perceived as competent in breastfeeding counseling. As seen in prior studies, programs with more maternity care exposure produce graduates who ultimately practice obstetric care, which likely shapes the readiness of graduates to provide lactation counseling. ${ }^{21}$ Though providers of prenatal care comprise $12 \%$ of practicing family physicians, many more family physicians will ultimately interface with lactation in their practice, through the pediatric ages or the postpartum period..$^{21,22}$

\section{Strengths}

This survey specifically targeted family medicine PDs, whereas recent lactation education research has typically grouped family medicine with other specialties. ${ }^{7,9,10} \mathrm{Re}-$ spondents to the survey were from geographically diverse regions of the country, with representation across the spectrum of rural to urban patient populations. The survey covered variables that are unique to family medicine training, including volume of prenatal care visits, and graduate $\mathrm{MCH}$ practice, but also surveyed variables that are relevant to pediatric and OB-GYN residencies, such as group prenatal care, BabyFriendly participation, education by lactation consultants, and the expectation for faculty assessment of resident counseling skills. This may inform targets for future research not only in family medicine, but also in other specialties.

\section{Limitations}

This study has several limitations. Results are limited by PDs' understanding of their MCH and breastfeeding curricula. Their subjective interpretation of resident competence may impart reporting and recall bias and social desirability. We would assume errors resulting from these biases would be uniformly distributed across respondents. The survey is cross-sectional so attributions of causation are limited to associations and hypothesis generation with the need for prospective testing of key curricular elements promoting breastfeeding competence. Moreover, the survey included the option of "unsure" for breastfeeding specific questions. This reduced the total pool of answers from which we were able to derive data, decreasing the power of our analyses. This also potentially excludes the answers of programs that have less lactation education, because these programs may also have PDs who are less confident in answering questions about their curricula. Furthermore, the survey does not include resident perspectives of their experience of breastfeeding education, and there may be a discrepancy between program director perceptions and resident selfperceived competence.

\section{Implications for Future Practice}

Despite its known public health benefit, current research in breastfeeding education is lacking, and specific research in family medicine residency departments lags behind other specialties. This study identifies characteristics of programs that have more breastfeeding education and resident competence in this important health-promoting skill. Some of the studied characteristics are less modifiable, such as academic affiliation, $\mathrm{MCH}$ visits in resident continuity clinic, and $\mathrm{MCH}$ practice among graduates. However, some factors that we identified may be gradually introduced to strengthen resident education and skills in breastfeeding counseling. Inclusion of lactation consultants as integral members of clinical staff, faculty engagement in assessing resident skills, and the implementation of group prenatal care models, are associated with resident competence in breastfeeding counseling. These represent targets for future research in our specialty, and opportunities for engaging family medicine residents in models of care that promote community health.

ACKNOWLEDGMENTS: The authors acknowledge Amy Cunningham, $\mathrm{PhD}$, Thomas Jefferson University Hospital, Department of Family Medicine, for her assistance in editing the survey questions.

CORRESPONDING AUTHOR: Address correspondence to Dr Zeynep Uzumcu, 833 Chestnut St, Suite 301, Philadelphia, PA 19107. Fax: 215-955-8600. uzumcu.zeynep@gmail.com.

\section{References}

1. American Academy of Family Physicians. Breastfeeding, Family Physicians Supporting (Position Paper). https://www.aafp.org/about/ policies/all/breastfeeding-support.html. Accessed September 8, 2019.

2. Johnston M, Landers S, Noble L, Szucs K, Viehmann L; Section on Breastfeeding. Breastfeeding and the use of human milk. Pediatrics. 2012;129(3):e827-e841.

3. U.S. Preventive Services Task Force. Primary care interventions to promote breastfeeding: recommendation statement. Am Fam Physician. 2010; 81(10),1265-1267.

4. Office of Disease Prevention and Health Promotion (US). Maternal, Infant, and Child Health: Objectives, Infant care. https://www. healthypeople.gov/2020/leading-healthindicators/2020-lhi-topics/Maternal-Infant-andChild-Health/determinants. Published 2010. Accessed May 21, 2020.

5. World Health Organization. UNICEF, WHA global nutrition targets 2025: breastfeeding policy brief. Geneva: WHO; 2014. 
6. Office of the Surgeon General (US); Centers for Disease Control and Prevention (US); Office on Women's Health (US). The Surgeon General's Call to Action to Support Breastfeeding. Rockville (MD): Office of the Surgeon General (US); 2011. A Call to Action. https://www.ncbi. nlm.nih.gov/books/NBK52691/. Accessed May $21,2020$.

7. Freed GL, Clark SJ, Sorenson J, Lohr JA Cefalo R, Curtis P. National assessment of physicians' breast-feeding knowledge, attitudes, training, and experience. JAMA. 1995;273(6):472-476.

8. Freed GL, Clark SJ, Curtis P, Sorenson JR. Breast-feeding education and practice in family medicine. J Fam Pract. 1995;40(3):263-269.

9. Ogburn T, Espey E, Leeman L, Alvarez K. A breastfeeding curriculum for residents and medical students: a multidisciplinary approach. J Hum Lact. 2005;21(4):458-464.

10. Feldman-Winter L, Barone L, Milcarek B, et al. Residency curriculum improves breastfeeding care. Pediatrics. 2010;126(2):289-297.

11. Rodriguez Lien E, Shattuck K. Breastfeeding Education and Support Services Provided to Family Medicine and Obstetrics-Gynecology Residents. Breastfeed Med. 2017;12(9):548-553.

12. World Health Organization; UNICEF. BabyFriendly Hospital Initiative. Revised, Updated and Expanded for Integrated Care. Geneva: World Health Organization; 2009.
13. Garretto D, Bernstein PS. CenteringPregnancy: an innovative approach to prenatal care delivery. Am J Obstet Gynecol. 2014;210(1):14-15.

14. Saenz RB. A lactation management rotation for family medicine residents. J Hum Lact. 2000;16(4):342-345

15. Sanchez A, Farahi N, Flower KB, Page CP. Improved Breastfeeding Outcomes Following an On-site Support Intervention in an Academic Family Medicine Center. Fam Med. 2019;51(10):836-840.

16. Centers for Disease Control and Prevention (US). National Immunization Survey: Breastfeeding Rates. https://www.cdc.gov/breastfeeding/data/nis_data/results.html. Accessed September 8, 2019 .

17. Sutter MB, Prasad R, Roberts MB, Magee SR. Teaching maternity care in family medicine residencies: what factors predict graduate continuation of obstetrics? A 2013 CERA program directors study. Fam Med. 2015;47(6):459-465.

18. Mainous AG III, Seehusen D, Shokar N. CAFM Educational Research Alliance (CERA) 2011 Residency Director survey: background, methods, and respondent characteristics. Fam Med. 2012;44(10):691-693.

19. 2019 Residency Program Directors (PD15 Survey Results. Leawood, KS: Society of Teachers of Family Medicine; 2019. https:// www.stfm.org/publicationsresearch/cera/ pasttopicsanddata/2019-residency-programdirectors-pd15-survey/. Accessed March 1, 2020
20. Bass J, Gartley T, Kleinman R, Bass J. Outcomes from the Centers for Disease Control and Prevention 2018 Breastfeeding Report Card: Public Policy Implications. J Pediatr. 2019.

21. Tong ST, Hochheimer CJ, Barr WB, et al. Characteristics of Graduating Family Medicine Residents Who Intend to Practice Maternity Care. Fam Med. 50(5):345-352.

22. Makaroff LA, Xierali IM, Petterson SM, Shipman SA, Puffer JC, Bazemore AW. Factors influencing family physicians' contribution to the child health care workforce. Ann Fam Med. 2014;12(5):427-431. 